\title{
On the downlink throughput capacity of hybrid wireless networks with massive MIMO
}

\author{
Baoju Zhang ${ }^{*}$ D, Yipeng Tian and Wei Wang
}

\begin{abstract}
In this paper, the entire network model is a hybrid wireless network model in which each base station is connected to each other via a wired link. On this basis, we place a large number of antennas (massive MIMO) at each base station to serve a single terminal in the downlink scenario, and we research the outage capacity and ergodic capacity in this scenario. The result of this paper is that the expressions for ergodic throughput capacity, outage probability, and outage throughput capacity have been derived under the favorable propagation condition. Through simulation, we can see the trend of outage throughput capacity and ergodic throughput capacity.
\end{abstract}

Keywords: Hybrid wireless network, Massive MIMO, Outage capacity, Ergodic capacity, Favorable propagation condition

\section{Introduction}

Because of the decentralized nature of ad hoc networks, nodes can join the network flexibly, but the problem with ad hoc networks have become apparent over long distances transmission due to lack of the infrastructure support. In this regard, in order to better combine both ad hoc and infrastructure advantages, hybrid wireless networks came into being [1]. In [2], Gupta and Kumar initiated the research on scaling law of large self-organizing networks and proposed the concept of scaling law. This research also promoted the research on the throughput capacity of hybrid wireless networks. In [3], T. L. Marzetta initiated the research of massive MIMO and found that using a wireless antenna system at the base station can completely eliminate the effects of uncorrelated receiver noise and fast fading, and the transmission of terminals within its own cell is not affected. In [4], the scaling law of throughput capacity of hybrid wireless network based on Nakagami-m fading channel is studied. In the uplink transmission phase, an optimal multiple access technique allows opportunistic sources to transmit concurrently with the scheduled source, and successive interference cancellation (SIC) strategy is then applied at the receiver to limit the intra-cell interference. However, in the downlink

\footnotetext{
* Correspondence: wdxyzbj@163.com

Tianjin Key Laboratory of Wireless Mobile Communications and Power Transmission, Tianjin Normal University, Tianjin 300387, China
}

transmission phase, neither of the two technologies in the uplink transmission phase can play a role, only one antenna is located at the base station and the receiving terminal respectively. In order to break the bottleneck of throughput capacity in the downlink transmission scenario in [4], the authors placed a point-to-point MIMO system on the base station [5]. In [6], the author analyzed the uplink outage throughput capacity of hybrid wireless networks with massive MIMO under the favorable propagation condition, where the $\mathrm{K}$ terminals wrap around the base station at the same distance. According to [5, 6], Massive MIMO can fully address the shortcomings of point-to-point MIMO, and under the favorable propagation condition, massive MIMO greatly mitigates the small scale-fading effect. So we think of throughput capacity change when the base station is equipped with a large number of antennas (massive MIMO), downlink services to a single terminal under the favorable propagation condition.

The rest of the paper is arranged as follows. In Section 2, we introduce the established hybrid wireless network model. In Section 3, we get the approximate rate of downlink transmission with massive MIMO under the favorable propagation condition. In Section 4 and Section 5, we obtain the expressions of outage probability, outage capacity, and ergodic capacity, respectively. In Section 6, the whole paper is summarized. 


\section{Hybrid wireless network model}

In order to facilitate the following analysis, we introduce the hybrid wireless network model as follows:

1. The entire network consists of $b$ base stations and $n$ nodes, each cell is assigned a base station, and each base station is placed in the center of the cell; $n$ nodes are evenly distributed in $b$ cells; the distance between parallel edges of each cell is $c=\sqrt{\frac{2 \sqrt{3}}{3}} \frac{n}{b}$.

2. The paper [7] shows that in order to obtain the gains provided by the infrastructure, $b=O\left(\frac{n}{\log n}\right)$ is the most basic condition.

3. We assume that the number of nodes in each cell is limited to $K$, and the proof method of $K$ in the square model is provided in [8]; by the approximation of this method, we can also prove that the number of nodes in the hexagon model cell is $K=\Theta\left(\frac{n}{b}\right)$.

4. We analyze the throughput capacity in the downlink transmission scenario; a large number of antennas (massive MIMO) at the base station send data to a single receiving terminal in the cell at the same time.

The hybrid wireless network model is shown in Fig. 1.

\section{Downlink asymptotic rate with massive MIMO}

In [9], when the number of antennas $M$ on the base station is much larger than the number of receiving terminals $K$, we call such a transmission mode the most favorable propagation condition. Under the most favorable propagation condition, the column vectors of the propagation matrix are asymptotically orthogonal,

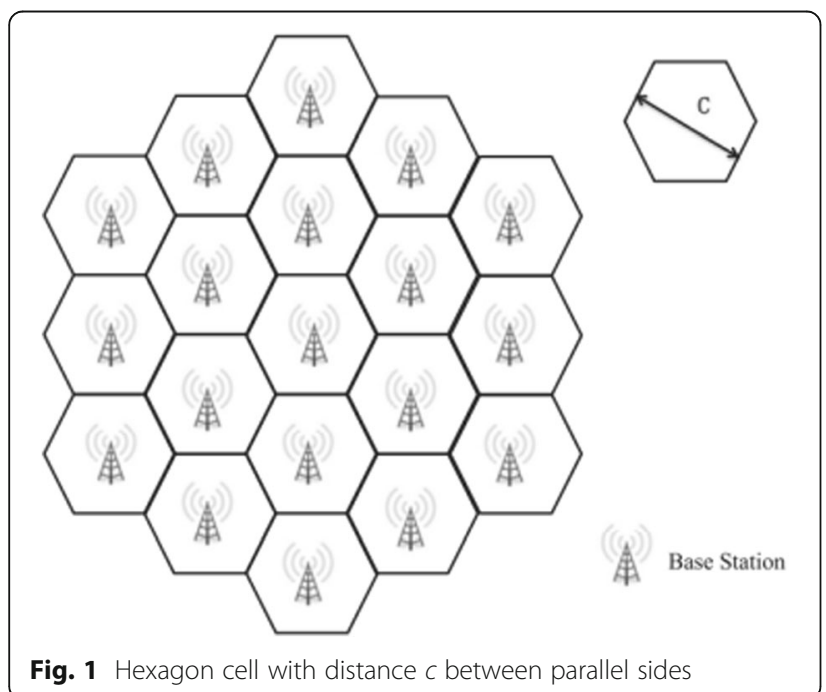

$$
\left(\frac{G^{H} G}{M}\right)_{M>>K}=D_{\beta}^{\frac{1}{2}}\left(\frac{H^{H} H}{M}\right)_{M>>K} D_{\beta}^{\frac{1}{2}} \approx D_{\beta}
$$

where $G$ represents the $M \times K$ propagation matrix, which consists of a small-scale fading matrix $H$ and a large-scale fading diagonal matrix $D_{\beta}^{\frac{1}{2}}$ (the $H$ in the upper right corner represents the conjugate transpose).

In the downlink transmission, the propagation matrix $G^{T}$ is the transpose of the uplink propagation matrix $G$, and the downlink model can be expressed as

$$
x_{d}=\sqrt{\rho_{d}} G^{T} s_{d}+N_{d}
$$

where $s_{d}$ is an $M \times 1$ vector transmitted by the $M$ antennas, $x_{d}$ is a $K \times 1$ vector received by $K$ terminals, $N_{d}$ is the noise vector whose components are independent and distributed with $N_{d} \sim C N(0,1)$, and $\rho_{d}$ is proportional to SNR. The total power in the downlink transmission is independent of the number of antennas; to facilitate the analysis, we assume the total power is one

$$
E\left\{\left\|s_{d}\right\|^{2}\right\}=1
$$

When both the base station and the receiving terminals have known the information of channel state, the base station serves $K$ terminals in the downlink transmission. In order to obtain the sum capacity, we need to make a constrained optimization first,

$$
\begin{aligned}
& C_{\text {sum-d }}=\max _{\left\{p_{k}\right\}} \log _{2} \operatorname{det}\left(I_{M}+\rho_{d} G D_{p} G^{H}\right) \\
& \sum_{k=1}^{K} p_{k}=1, p_{k} \geq 0
\end{aligned}
$$

where $D_{p}$ is a diagonal matrix whose diagonal elements constitute a $K \times 1$ vector $p$. The power limit is given by Eq. (5), and the sum power is limited to 1 . In the case of satisfying the best power of each terminal, we can get the sum rate under the favorable propagation condition as

$$
\begin{aligned}
& C_{\text {sum }-d-M>>K}=\max _{\left\{p_{k}\right\}} \log _{2} \operatorname{det}\left(I_{K}+\rho_{d} D_{p}^{\frac{1}{2}} G^{H} G D_{p}^{\frac{1}{2}}\right) \\
& C_{\text {sum }-d-M>>K}=\sum_{k=1}^{K} \log _{2}\left(1+M \rho_{d} p_{k} \beta_{k}\right)
\end{aligned}
$$

where $p_{k}$ represents the optimal power allocated for each node after power constraint optimization, and $\beta_{k}$ represents the large-scale fading coefficient. According to [10], we can define $\beta_{k}$ with the following equation

$$
\beta_{k}=\phi d_{k}^{-\alpha} \zeta_{k}
$$

where $\phi$ is a constant related to the antenna gain and carrier frequency, $d_{k}$ is the distance between the base station 
and $k^{\text {th }}$ terminal, $\alpha$ is the path loss exponent, and $\zeta_{k}$ is the log-normal shadowing with $10 \log _{10} \zeta_{k} \sim N\left(0, \sigma_{k}^{2}\right)$.

According to the above analysis, in order to achieve the purpose of this paper, we can get the throughput capacity expression by combining Eq. (7) and Eq. (8). So, the throughput capacity expression is

$$
C=\log _{2}\left(1+M \rho_{d} \phi d^{-\alpha} \zeta\right)
$$

\section{Outage probability and outage capacity}

The outage capacity is the maximum transmission rate that the system can achieve for a given outage probability [11]. In [4], in the downlink transmission phase, neither the optimal multiple access technique nor successive interference cancellation (SIC) strategy is allowed, each node outage capacity over Nakagami-m fading channel scales as $\Theta\left(\frac{b}{n} \log \left(\varepsilon^{\frac{1}{m}} \frac{n}{b}\right) W_{2}\right)$ under infrastructure mode. Applying massive MIMO to hybrid wireless networks, the channel capacity under infrastructure mode will be significantly different from that in $[4,5]$.

According to our above analysis, we can define the outage probability as

$$
P_{\text {out }}=\operatorname{Pr}\left\{\log _{2}\left(1+M \rho_{d} \phi d^{-\alpha} \zeta\right)<R\right\}
$$

In the case of low SNR, we use the approximation $\ln (1+x) \approx x$ to calculate the outage probability as follows

$$
P_{\text {out }}=\operatorname{Pr}\left\{\log _{2}^{e} M \rho_{d} \phi d^{-\alpha} \zeta<R\right\}
$$

We define a new variable $T$, where the variable $T=d^{-\alpha} \zeta$

$$
P_{\text {out }}=\operatorname{Pr}\left\{T<\frac{R}{\log _{2}^{e} M \rho_{d} \phi}\right\}
$$

The shadow fading $\zeta$ satisfies the log-normal distribution with $10 \log _{10} \zeta \sim N\left(0, \sigma^{2}\right)$, so the variable $T$ we defined is also satisfied with the log-normal distribution with $10 \log _{10} T \sim N\left(\mu, \sigma^{2}\right)$ and $\mu=10 \log _{10} d^{-\alpha}$. According to the log-normal probability density function (pdf), we can get the outage probability as

$$
\begin{aligned}
& P_{\text {out }}=\int_{0}^{S} \frac{10}{\ln 10 \sqrt{2 \pi} \sigma T} e^{-\frac{\left(1 \log _{10} T-\mu\right)^{2}}{2 \sigma^{2}}} d T \\
& S=\frac{R}{\log _{2}^{e} M \rho_{d} \phi}
\end{aligned}
$$

According to the nature of the log-normal distribution, the outage probability is represented by the complementary error function as

$$
P_{\text {out }}=\frac{1}{2} \operatorname{erfc}\left(-\frac{10 \log _{10} S-\mu}{\sigma \sqrt{2}}\right)
$$

In [12], we can get a tight exponential upper bound of the complementary error function and a pure exponential approximation for the complementary error function, as follows

$$
\begin{aligned}
& \operatorname{erfc}(x) \approx \frac{1}{6} e^{-x^{2}}+\frac{1}{2} e^{-\frac{4}{3} x^{2}}, x>0 \\
& \operatorname{erfc}(x) \leq \frac{1}{2} e^{-2 x^{2}}+\frac{1}{2} e^{-x^{2}} \leq e^{-x^{2}}, x>0
\end{aligned}
$$

The precondition for obtaining the outage capacity is $P_{\text {out }}=\varepsilon$. In the approximation by Eq. (16), we create a new variable $S^{\prime}$ and $S^{\prime}=e^{-\left(-\frac{10 \log _{10} S-\mu}{\sigma \sqrt{2}}\right) 2}$. Substituting $S^{\prime}$ to Eq. (17), the upper bound of the outage probability we can get is

$$
\begin{aligned}
& P_{\text {out }} \leq \frac{1}{2}\left(\frac{1}{2}\left(S^{\prime}\right)^{2}+\frac{1}{2} S^{\prime}\right)=\varepsilon \\
& S^{\prime}=\frac{-1+\sqrt{1+16 \varepsilon}}{2}
\end{aligned}
$$

When $\varepsilon<\frac{1}{2}$, Eq. (19) is satisfied. Based on the above analysis, we can get the outage throughput capacity expression at low SNR as follows

$$
C_{\varepsilon}=\log _{2}^{e} M \rho_{d} \phi 10 \frac{\mu-\sigma \sqrt{2 \ln \left(\frac{2}{-1+\sqrt{1+16 \varepsilon}}\right)}}{10}
$$

Since the scalar $\rho_{d}$ is proportional to SNR, in the following simulation, we consider the change of outage capacity with $\rho_{d}$.

Simulation results are shown in Fig. 2.

In the case of high SNR, we use the approximation $\log (1+x)=\log (x)$ to calculate the outage probability as follows

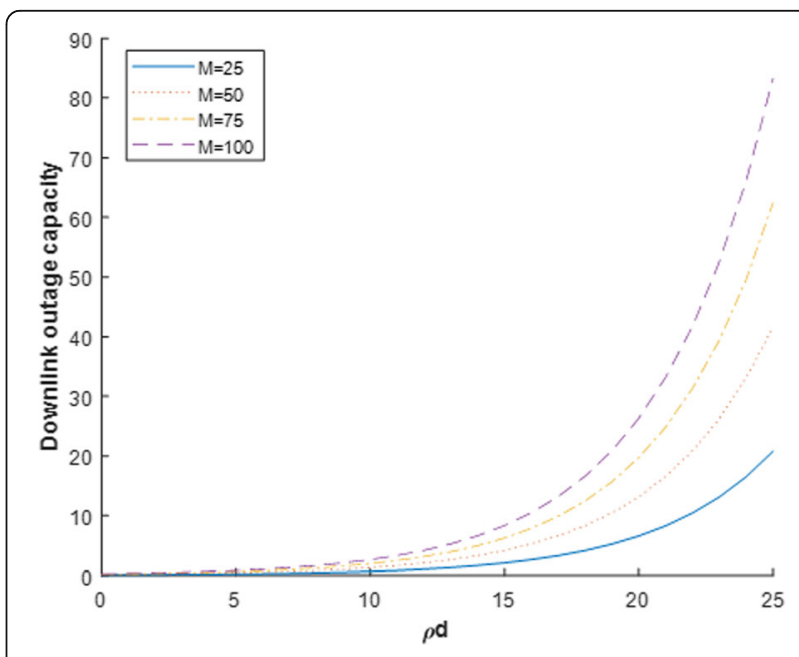

Fig. 2 Downlink outage capacity at low SNR 


$$
P_{\text {out }}=\operatorname{Pr}\left\{\log _{2}\left(M \rho_{d} \phi d^{-\alpha} \zeta\right)<R\right\}
$$

Here, we define a new variable $X$ and $X=\log _{2}\left(M \rho_{d} \phi d^{-\alpha} \zeta\right)$. The variable $\zeta$ obeys the log-normal distribution with $10 \log _{10} \zeta \sim N\left(0, \sigma^{2}\right)$. By changing the formula for logarithms and a series of calculations, we find that the new variable $X$ is a random variable that satisfies the normal distribution with $X \sim N\left(\mu_{X}, \sigma_{X}^{2}\right)$. The values of $\mu_{X}$ and $\sigma_{X}$ are respectively as follows

$$
\begin{aligned}
& \mu_{X}=\log _{2}\left(M \rho_{d} \phi d^{-\alpha}\right) \\
& \sigma_{X}^{2}=\left(\frac{\sigma}{10 \log _{10} 2}\right)^{2}
\end{aligned}
$$

Similarly, in the way we analyzed before, we can get the following equations of outage probability

$$
\begin{aligned}
& P_{\text {out }}=\operatorname{Pr}\{X<R\} \\
& P_{\text {out }}=\varepsilon=\frac{1}{2} \operatorname{erfc}\left(-\frac{R-\mu_{X}}{\sigma_{X} \sqrt{2}}\right)
\end{aligned}
$$

Equation (25) is satisfied when $\varepsilon<\frac{1}{2}$, so we can get the outage throughput capacity expression at high SNR:

$$
C_{\varepsilon}=\mu_{X}-\sigma_{X} \sqrt{2 \ln \left(\frac{2}{-1+\sqrt{1+16 \varepsilon}}\right)}
$$

By combining Eq. (22), Eq. (23), and Eq. (26), we can get

$$
C_{\varepsilon}=\log _{2}\left(M \rho_{d} \phi d^{-\alpha}\right)-\left(\frac{\sigma}{10 \log _{10} 2}\right) \sqrt{2 \ln \left(\frac{2}{-1+\sqrt{1+16 \varepsilon}}\right)}
$$

Simulation results are shown in Fig. 3.

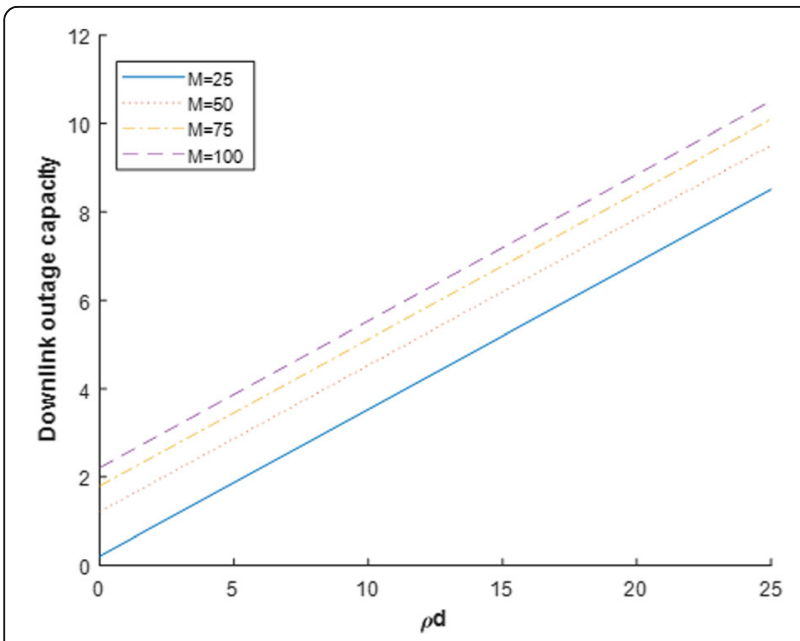

Fig. 3 Downlink outage capacity at high SNR

\section{Ergodic capacity}

Ergodic capacity refers to the time average of the maximum information rate of random channels in all fading states [13]. In [14], in the downlink transmission phase, the successive interference cancellation strategy (SIC) is not feasible, the per-node ergodic throughput capacity over Nakagami-m fading channel at low SINR scales as $\Theta\left(W_{2}\right)$ under the infrastructure transmission mode, and the per-node ergodic throughput capacity at high SINR is $\Theta\left(\frac{b}{n} \log \left(\frac{1}{m} \cdot \frac{n}{b}\right) W_{2}\right)$ ( $W_{2}$ is the bandwidth allocated for the downlink transmission; $m$ is the shape parameter). In [15], the change of ergodic throughput capacity under the infrastructure transmission mode with distributed base stations (DBS) is analyzed. The ergodic capacity gain of the hybrid wireless network with distributed base stations is $N \times N_{B S}$ that compared to the traditional hybrid wireless network ( $N$ is the number of distributed base stations; $N_{\mathrm{BS}}$ is the number of antennas placed at the base station). Based on the previous analysis, what will happen to the ergodic throughput capacity under the infrastructure transmission mode when a large number of antennas (massive MIMO) are placed at the base station?

According to the above Eq. (9), we define $\bar{C}$ on behalf of the ergodic capacity; the expression of the downlink ergodic capacity is as follows

$$
\bar{C}=E\left[\log _{2}\left(1+M \rho_{d} \phi d^{-\alpha} \zeta\right)\right]
$$

Similarly, in the previous analysis, at low SNR, we approximate $\ln (1+x) \approx x$, then we can get the expression of the ergodic capacity as

$$
\bar{C}=E\left[\log _{2}^{e} M \rho_{d} \phi W\right]
$$

where $W$ is the same as the variable $T$ defined in Eq. (12) above. According to the means of averaging, we can get

$$
E(W)=\frac{10}{\sqrt{2 \pi} \sigma \ln 10} \int_{-\infty}^{+\infty} e^{\frac{(10 \lg W-\mu)^{2}}{2 \sigma^{2}}} d W
$$

By transforming $\frac{10 \lg W-\mu}{\sigma}$ to $Z$, making integral to the expression again, we can get

$$
E(W)=10^{\frac{\mu+10}{10}} e^{\frac{\sigma^{2}}{100}}
$$

Combining Eq. (29) and Eq. (31), the ergodic throughput capacity at low SNR is

$$
\bar{C}=\log _{2}^{e} M \rho_{d} \phi 10^{\frac{\mu+10}{10}} e^{\frac{\sigma^{2}}{100}}
$$

Simulation results are shown in Fig. 4.

At high SNR, using the approximation $\log (1+x)=$ $\log (x)$, we can get the following ergodic throughput capacity expression 


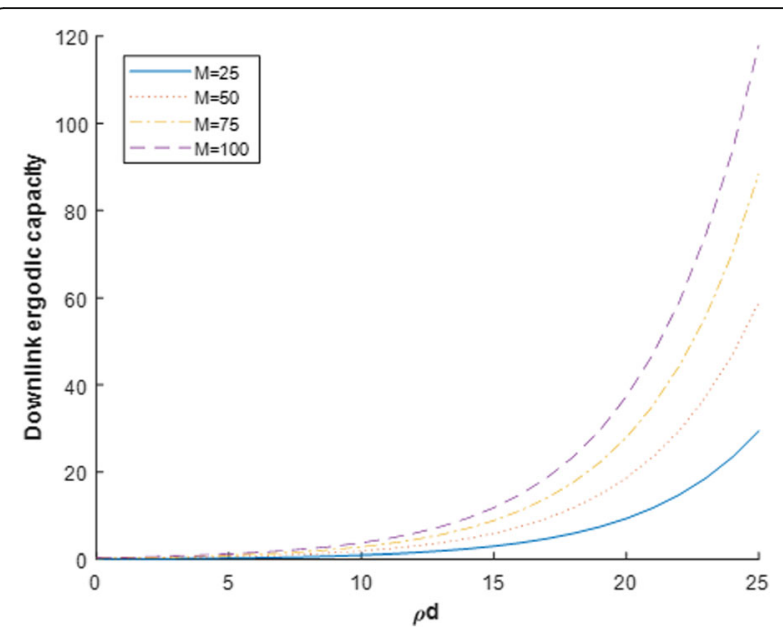

Fig. 4 Downlink ergodic capacity at low SNR

$$
\bar{C}=E\left[\log _{2}\left(M \rho_{d} \phi d^{-\alpha} \zeta\right)\right]
$$

As with the method of analyzing the outage capacity at high SNR, we also define $\log _{2}\left(M \rho_{d} \phi d^{-\alpha} \zeta\right)$ as the variable $X$ above, we can get

$$
\bar{C}=\mu_{X}=\log _{2}\left(M \rho_{d} \phi d^{-\alpha}\right)
$$

Simulation results are shown in Fig. 5.

\section{Conclusions}

In this paper, we mainly analyzed the downlink transmission throughput capacity of the hybrid wireless network with massive MIMO under the favorable propagation condition. Under the favorable propagation condition, the column vectors of the propagation matrix are asymptotically

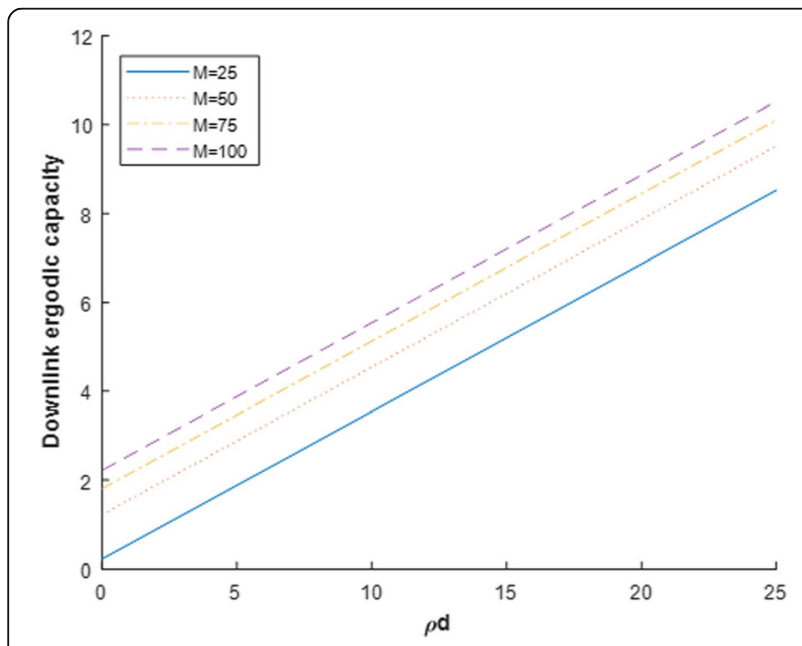

Fig. 5 Downlink ergodic capacity at highSNR orthogonal, and large-scale fading became the dominant factor. From the above analysis, we derived the closed expressions of the outage throughput capacity and the ergodic throughput capacity (low SNR and high SNR) in the downlink transmission scenario when the base station is placed with massive MIMO. With the help of simulation, we can find that when the number of antennas $M$ at the base station increase, the outage throughput capacity and the ergodic throughput capacity also increase, but the extent of growth is different.

\section{Funding}

This paper is supported by the Natural Science Foundation of China (61271411) and Natural Youth Science Foundation of China (61501326, 61401310). It is also supported by the Tianjin Research Program of Application Foundation and Advanced Technology (15JCZDJC31500) and Tianjin Science Foundation (16JCYBJC16500).

\section{Authors' contributions}

BZ gives the overall research direction and ideas. YT read the relevant literature and books and drafts the article. WW makes the corresponding experimental simulation. All authors read and approved the final manuscript.

\section{Authors' information}

Baoju Zhang is a professor at College of Electronic and Communication Engineering of Tianjin Normal University. She received the B.S. degree from Tianjin Normal University in 1990, M.S. degree from Tianjin Normal University in 1993, and Ph.D. degree from Tianjin University in 2002. Her main research directions involve compressive sensing, audio and video processing, data stream clustering, and so on.

Yipeng Tian is working on his M.S. degree in Electronics and Communication Engineering at Institute of Tianjin Normal University; he received his B.S. degree in Tianjin Normal University. He is studying hybrid wireless network and massive MIMO capacity.

Wei Wang received his Ph.D. degree in Tianjin University; currently, he is an Associate Professor in College of Electronic and Communication Engineering, Tianjin Normal University, Tianjin, China. His main research directions involve compressive sensing, radar signal processing, optical fiber sensing technology and applications, and so on.

\section{Competing interests}

The authors declare that they have no competing interests.

\section{Publisher's Note}

Springer Nature remains neutral with regard to jurisdictional claims in published maps and institutional affiliations.

Received: 5 March 2018 Accepted: 26 April 2018

Published online: 08 May 2018

\section{References}

1. O Dousse, P Thiran, M Hasler, Connectivity in ad-hoc and hybrid networks. Proceedings - IEEE INFOCOM, IEEE: Institute of Electrical and Electronics Engineers Inc. 2, 1079-1088, (2002). https://doi.org/10.1109//NFCOM.2002. 1019356

2. P Gupta, PR Kumar, The capacity of wireless networks. IEEE Trans. Inf. Theory 46(2), 388-404 (2000)

3. TL Marzetta, Noncooperative cellular wireless with unlimited numbers of base station antennas. IEEE Trans. Wirel. Commun. 9(11), 3590-3600 (2010)

4. X Wang, Q Liang, in Proc. IEEE Global Commun. Conf. On the outage throughput capacity of hybrid wireless networks over fading channels (2012), pp. 2173-2178

5. B Zhang, Y Wang, W Wang, Y Tian, On the downlink throughput capacity of hybrid wireless networks with MIMO. IEEE Access 5, 26086-26091 (2017)

6. G Zhao, Q Liang, On the uplink outage throughput capacity of hybrid wireless networks with massive MIMO. Ad Hoc Netw. 58, 62-69 (2017)

7. B Liu, Z Liu, D Towsley, On the capacity of hybrid wireless networks. Info 2, 1543-1552 (2003) 
8. D Kirachaiwanich, Q Liang, Capacity of wireless hybrid networks with successive interference cancellation. IEEE Global Telecommunications Conference: Institute of Electrical and Electronics Engineers Inc. (2010). https://doi.org/10.1109/GLOCOM.2010.5684015

9. F Rusek et al., Scaling up MIMO: opportunities and challenges with very large arrays. IEEE Signal Process. Mag. 30(1), 40-60 (2013)

10. K Zheng, $\mathrm{S} O \mathrm{Ou}, \mathrm{X}$ Yin, Massive MIMO channel models: a survey. Int. J. Antennas Propag. 2014, 10 (2014) Article ID 848071

11. L Ozarow, S Shamai, A Wyner, Information-theoretic considerations in cellular mobile radio. IEEE Trans. Veh. Technol. 43, 359-378 (1994-05)

12. M Chiani, D Dardari, MK Simon, New exponential bounds and approximations for the computation of error probability in fading channels. IEEE Trans. Wirel. Commun. 2(4), 840-845 (2003)

13. JG Proaksi, M Salehi, in Digtial communication, ed. by 5th. (McGraw-Hill Education, 2008)

14. X Wang, Q Liang, On the throughput capacity and performance analysis of hybrid wireless networks over fading channels. IEEE Trans. Wirel. Commun. 12(6), 2930-2940 (2013)

15. Z Li, Q Liang, in IEEE Glob. Commun. Conf., GLOBECOM. Scaling laws for the ergodic capacity of hybrid wireless networks with distributed base stations (2015)

\section{Submit your manuscript to a SpringerOpen ${ }^{\circ}$ journal and benefit from:}

- Convenient online submission

Rigorous peer review

- Open access: articles freely available online

- High visibility within the field

- Retaining the copyright to your article

Submit your next manuscript at $\boldsymbol{s p r i n g e r o p e n . c o m ~}$ 